\section{Photodynamic therapy combined with posterior subtenon triamcinolone acetonide injection in the treatment of choroidal neovascularization}

Y-A Lee, T-C Ho, M-S Chen, C-H Yang and C-M Yang

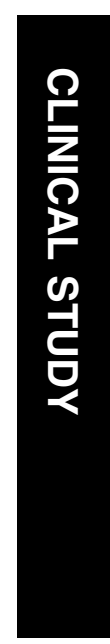

\begin{abstract}
Purpose To evaluate the therapeutic effect of photodynamic therapy (PDT) combined with posterior subtenon injection of triamcinolone acetonide (PSTA) in the treatment of choroidal neovascularization (CNV).

Methods In this retrospective case-control study, treatment outcomes at 12 months of patients with $\mathrm{CNV}$ were reviewed. One hundred and two consecutive patients (102 eyes) with subfoveal CNV of various causes were included. Patients in the study group $(n=46)$ received PDT followed within 2 days by $40 \mathrm{mg}$ of PSTA. Patients in the control group $(n=56)$ received PDT alone. Patients were retreated 3 months later or more if fluorescein angiography showed residual membrane leakage. Treatment outcomes were compared between the two groups and among different CNV subgroups.

Results Gain in mean best-corrected visual acuity (BCVA) was significantly higher in the study group $(+0.78$ lines) than in the control group $(-1.86$ lines $)(P<0.0001)$. The number of treatments within 1 year was significantly less in the study group (1.26 vs $1.63, P=0.008)$. The mean BCVA change for myopic CNV and age-related predominantly classic CNV subgroups was significantly higher in the study group $(+2.82$ vs -0.91 lines, $P=0.0005$ for myopic CNV; +0.6 vs -1.79 lines, $P=0.01$ for age-related predominantly classic $\mathrm{CNV}$ ). The main side effect in the study group was increased intraocular pressure $(8.7 \%)$.
\end{abstract}

Conclusion Compared with PDT alone, PDT combined with PSTA has a better therapeutic effect for both myopic and age-related predominantly classic $\mathrm{CNV}$; the myopic $\mathrm{CNV}$ subgroup shows the best response.

Eye (2009) 23, 645-651; doi:10.1038/eye.2008.17; published online 22 February 2008

Keywords: photodynamic therapy; triamcinolone acetonide; choroidal neovascularization; posterior subtenon injection

Introduction

Choroidal neovascularization $(\mathrm{CNV})$ is an important pathologic change seen in a variety of chorioretinal diseases. ${ }^{1}$ Advanced age-related macular disease (AMD) is the leading cause of irreversible central vision loss in older adults in the western world. Of the various types of AMD causing severe vision loss, approximately $80-90 \%$ of cases involve the neovascular form. ${ }^{2,3}$ $\mathrm{CNV}$ is also a common cause of vision loss in certain groups of patients younger than 50 years of age. ${ }^{4}$ In high myopic patients, $\mathrm{CNV}$, in addition to retinal detachment, is the most vision-threatening complication., 5 This lesion is particularly important in Asian countries because of the high incidence of high myopia on that continent (9-21\%). ${ }^{7,8}$

Photodynamic therapy (PDT) with verteporfin has been shown to safely reduce the risk of severe vision loss in patients with subfoveal CNV secondary to AMD. ${ }^{9,10}$ It has
Department of Ophthalmology, National Taiwan University Hospital, Taipei, Taiwan

Correspondence: C-M Yang, Department of Ophthalmology، National Taiwan University Hospital and School of Medicine, National Taiwan University, No. 7 Chung-Shan South Road, Taipei 100, Taiwan. Tel: + 886223123456 , ext. 5187:

Fax: + 886223934420

E-mail: chungmay@

ntu.edu.tw

Received: 4 July 2007 Accepted in revised form: 14 January 2008 Published online: 22 February 2008 
also been reported to be an effective treatment for subfoveal CNV caused by pathologic myopia. ${ }^{11}$ However, CNV regrowth is common and repeated PDT is needed to prevent further vision loss. ${ }^{12}$ The surge of local angiogenic factors caused by choroidal hypoperfusion, persistent inflammation from CNV itself, and following treatment may also contribute to the high rate of recurrence. ${ }^{13,14}$

Several studies have shown that PDT combined with intravitreal injection of the corticosteroid triamcinolone acetonide (IVTA) is more effective for treating CNV than PDT alone. ${ }^{15-19}$ However, IVTA has been shown to produce side effects, such as cataract progression, elevated intraocular pressure (IOP), endophthalmitis, and retinal detachment. ${ }^{20}$ Alternatively, posterior subtenon injection of triamcinolone acetonide (PSTA) also has anti-inflammatory effects and has been found to be effective for classic CNV secondary to AMD. ${ }^{21,22}$ Furthermore, PSTA has fewer side effects than IVTA. ${ }^{23}$ In addition, subtenon injection of depo forms of corticosteroids may last considerably longer than that of intravitreal injections. We hypothesize that PSTA combined with PDT may increase the therapeutic effect of PDT, and lead to fewer side effects than IVTA. In this study, we investigate the therapeutic effect and safety of PDT combined with PSTA to treat different types of subfoveal CNV.

\section{Materials and methods}

This retrospective, case-control study was approved by the Institutional Review Board of National Taiwan University Hospital. Patients with various types of subfoveal CNV treated with PDT alone or PDT combined with PSTA at National Taiwan University Hospital from January 2004 to July 2006 were included in this study. Indications for PDT were based on the results of TAP/ VIP studies. ${ }^{11,24}$ The inclusion criteria were CNV complex under geometric centre of the foveal avascular zone with greatest linear dimension of lesion $\leqslant 5400 \mu \mathrm{m}$, and an area of $\mathrm{CNV}$ composing at least $50 \%$ of the total neovascular lesion. The exclusion criteria included multiple haemorrhagic retinal pigment epithelial (RPE) detachment or the presence of subretinal orange nodular lesions indicative of polypoidal choroidal vasculopathy, tear of retinal pigment epithelium, active hepatitis or clinically significant liver disease, intraocular surgery within the last 2 months, porphyria or other porphyrin sensitivity, and inability to obtain photographs to document CNV. Indocyanin green angiography was done when the diagnosis of polypoidal choroidal vasculopathy was uncertain. Confirmed cases were excluded. Patients with other major ocular or systemic disease influencing vision were also excluded. The follow-up period was set at 1 year.

All patients received basic ophthalmic examinations, which included best-corrected visual acuity (BCVA) in logMAR scale, slit lamp biomicroscopy, and measurement of IOP. Fluorescein angiography (FA) was performed to identify the location and type of the $\mathrm{CNV}$ lesion, and to determine whether there was active leakage.

A total of 102 sequential patients (102 pair of eyes) receiving PDT in a standard manner described in the TAP study were included in our study. ${ }^{9}$ Four types of $\mathrm{CNV}$ were identified from the study cases:

predominantly classic CNV secondary to AMD, predominantly occult CNV secondary to AMD, myopic $\mathrm{CNV}$, and idiopathic CNV. Predominantly classic CNV is defined as well-demarcated CNV occupying more than $50 \%$ of the total neovascular lesion; predominantly occult $\mathrm{CNV}$ is defined as poorly demarcated CNV occupying more than $50 \%$ of the total neovascular lesion; myopic $\mathrm{CNV}$ was defined as $\mathrm{CNV}$ in a myopic eye of more than -6 dioptres spherical equivalent with some evidence of myopic degeneration changes; and idiopathic $\mathrm{CNV}$ is established by exclusion. Three ophthalmologists (CMY, $\mathrm{TCH}$, and $\mathrm{CHY}$ ) performed the treatment; one of them (CMY) routinely gave additional PSTA to patients ( $n=46$ ) within $48 \mathrm{~h}$ after PDT based on the physician's preference. The remaining patients $(n=56)$ had only the PDT procedure.

In the PSTA treatment, the triamcinolone acetonide $\left(40 \mathrm{mg}\right.$ ) was drawn into a $1-\mathrm{cm}^{3}$ syringe using a 23 -gauge needle, and the eye was anaesthetized with proparacaine $0.5 \%$. The needle was then changed to a 25-gauge, 0.5 -inch one, and the patient was asked to look superonasally with the lower eyelid held manually. The inferotemporal conjunctiva and tenon were penetrated with the needle with the bevel facing upward. To prevent engaging the sclera, the needle was rotated to the bevel-down position and moved from side to side while advancing before injection. After aspiration to avoid intravascular injection, $40 \mathrm{mg}$ of triamcinolone acetonide was injected into the posterior subtenon space. A cotton tip applicator was used to compress the lower temporal fornix while withdrawing the needle to prevent reflux. We chose the inferior route rather than the superior route for PSTA to avoid the complication of ptosis. ${ }^{25,26}$

All patients were followed at 3-month intervals. Follow-up examinations included measurement of BCVA, FA, measurement of IOP, and slit lamp biomicroscopy. If active CNV leakage was noted by FA, the patient received further PDT with PSTA (study group) or PDT alone (control group). We recorded the types and aetiologies of $\mathrm{CNV}$ in all patients. The side effects of treatment were also recorded. To evaluate the 
treatment effect of PDT with PSTA, we compared the overall 1-year BCVA, and the percentage of eyes with BCVA loss of more than three lines or increase of more than three lines between the two groups. The number of treatments and disease progression in the two groups were analysed by paired $t$-test using SAS version 9.0. These parameters were also compared among different CNV subgroups.

\section{Results}

The baseline data of all patients are presented in Table 1. There was no significant difference in age, sex, lesion size, or BCVA between patients in the study group and those in the control group (all $P>0.05$ ). Furthermore, there were no significant differences in type or aetiology of CNV between the two groups (Table 2). The most frequently seen type of CNV in this study was myopic $\mathrm{CNV}$, followed by classic or occult-type CNV secondary to AMD.

The mean BCVA change from baseline to 12 months after treatment in the study group was an improvement of 0.78 lines with a confidence interval of \pm 3.2 lines. The mean BCVA change in the control group was a loss of 1.86 lines with a confidence interval of \pm 3.15 lines. The difference of mean BCVA change between the two groups was significant $(P<0.0001)$. The average number of treatments was $1.26( \pm 0.57)$ in the study group and $1.63( \pm 0.75)$ in the control group. There is a significant difference between these two groups $(P=0.008)$. One year after treatment, two patients in the study group $(4.3 \%)$ and eight patients in the control group (14.3\%) still had active CNV lesions.
The mean BCVA change for eyes with myopic CNV was $+2.82( \pm 2.58)$ lines in the study group and $-0.91( \pm 3.31)$ lines in the control group. The mean BCVA change for eyes with age-related predominantly classic CNV was $+0.6( \pm 1.9)$ lines in the study group and $-1.79( \pm 2.33)$ lines in the control group. The differences between the study and the control groups were statistically significant in these two subgroups $(P=0.0005$ for myopic $\mathrm{CNV} ; P=0.01$ for age-related predominantly classic $\mathrm{CNV}$ ). The mean BCVA change for eyes with age-related predominantly occult $\mathrm{CNV}$ was $-1.75( \pm 3.39)$ lines in the study group and $-3.42( \pm 2.84)$ lines in the control group. The mean BCVA change for eyes with idiopathic $\mathrm{CNV}$ was $+0.43( \pm 2.76)$ lines in the study group and $-2.25( \pm 3.92)$ lines in the control group. The differences between the study and control groups were not significant in these two subgroups $(P=0.2$ for age-related predominantly occult $\mathrm{CNV}$ and $P=0.16$ for idiopathic CNV).

Table 3 shows the percentage of BCVA increase of more than three lines in each subgroup. Patients with myopic CNV had the highest percentage reaching this level of improvement among the four study subgroups, and showed the most favourable response to PDT + PSTA than to PDT alone in comparison with other subgroups (from $9.1 \%$ with PDT alone to $29.4 \%$ with PDT + PSTA). Patients with myopic CNV and with predominantly classic CNV secondary to AMD showed lower percentage of a BCVA loss of more than three lines in the study group than in the control group (Table 4).

Further subgroup analysis was done for myopic CNV, which appeared to benefit most from the combination treatment. The baseline characteristics between the study

Table 1 Baseline data of age, sex, lesion size, and BCVA in the study group and the control group

\begin{tabular}{lccc}
\hline & PDT + PSTA & PDT alone & P-value \\
\hline Age & $57.07( \pm 16.06)$ & $56.2( \pm 17.7)$ & 0.80 \\
Sex (male/female) & $20 / 26$ & $24 / 32$ & 0.95 \\
Right eye/left eye & $25 / 21$ & $30 / 26$ & 0.94 \\
Lesion size (im) & $1934.8( \pm 1080.8)$ & $1964.3( \pm 1192.6)$ & 0.90 \\
Baseline BCVA (logMAR) & $1.11( \pm 0.59)$ & $0.99( \pm 0.53)$ & 0.31 \\
\hline
\end{tabular}

$\mathrm{BCVA}=$ best-corrected visual acuity; logMAR = logarithm of the minimum angle of resolution; $\mathrm{PDT}=$ photodynamic therapy; $\mathrm{PSTA}=$ posterior subtenon injection of triamcinolone acetonide.

The results are expressed as mean \pm SD.

Table 2 Number of patients according to types of choroidal neovascularization in the study group and the control group

\begin{tabular}{lcccrc}
\hline & AMD, classic predominant & AMD, occult predominant & Myopic CNV & Idiopathic & Total \\
\hline PDT + PSTA & $10(21.7 \%)$ & $12(26.1 \%)$ & $17(37.0 \%)$ & $7(15.2 \%)$ & $46(100 \%)$ \\
PDT alone & $14(25 \%)$ & $12(21.4 \%)$ & $22(39.3 \%)$ & $8(14.3 \%)$ & $56(100 \%)$ \\
\hline
\end{tabular}

$\mathrm{AMD}=$ age-related macular degeneration; $\mathrm{CNV}=$ choroidal neovascularization; $\mathrm{PDT}=$ photodynamic therapy; PSTA $=$ posterior subtenon injection of triamcinolone acetonide.

$P$-value $>0.05$ in all types of choroidal neovascularization membrane between the study and control groups. 
group and the control group did not show statistical difference in terms of age (48.8 \pm 17.8 vs $50.2 \pm 18.3)$, gender (male/female, $9 / 8$ vs 10/12), mean spherical equivalent refractive error $(-10.2 \pm 3.1$ vs $-10.8 \pm 3.6 \mathrm{D})$, and mean greatest linear dimension of $\mathrm{CNV}$ $(1380.5 \pm 696.7$ vs $1317.8 \pm 731.6 \mu \mathrm{m})$ (all $P>0.05)$. The treatment outcomes are summarized in Table 5. The percentages of eyes with a BCVA increase of more than one line, two lines, or above were higher in the study group compared with the control group.

Table 3 Percentage of eyes with BCVA increase of more than three lines in the study group and the control group

\begin{tabular}{lcc}
\hline & $P D T+P S T A$ & PDT alone \\
\hline $\begin{array}{l}\text { Predominantly classic } \\
\text { CNV secondary to AMD }\end{array}$ & $1 / 10(10 \%)$ & $1 / 14(7.1 \%)$ \\
Predominantly occult & $0 / 12(0 \%)$ & $0 / 12(0 \%)$ \\
CNV secondary to AMD & & \\
Myopic CNV & $5 / 17(29.4 \%)$ & $2 / 22(9.1 \%)$ \\
Idiopathic CNV & $0 / 7(0 \%)$ & $0 / 8(0 \%)$ \\
Total & $7 / 46(15.2 \%)$ & $3 / 56(5.4 \%)$ \\
\hline
\end{tabular}

$\mathrm{AMD}=$ age-related macular degeneration; $\mathrm{BCVA}=$ best-corrected visual acuity; $\mathrm{CNV}=$ choroidal neovascularization; $\mathrm{PDT}=$ photodynamic therapy; PSTA $=$ posterior subtenon injection of triamcinolone acetonide.

Table 4 Percentage of eyes with BCVA loss of more than three lines in the study group and the control group

\begin{tabular}{lcc}
\hline & PDT + PSTA & PDT alone \\
\hline $\begin{array}{l}\text { Predominantly classic } \\
\text { CNV secondary to AMD }\end{array}$ & $1 / 10(10 \%)$ & $3 / 14(21.4 \%)$ \\
Predominantly occult & $2 / 12(16.7 \%)$ & $3 / 12(25 \%)$ \\
CNV secondary to AMD & & \\
Myopic CNV & $0 / 17(0 \%)$ & $2 / 22(9.1 \%)$ \\
Idiopathic CNV & $1 / 7(14.3 \%)$ & $1 / 8(12.5 \%)$ \\
Total & $4 / 46(8.7 \%)$ & $9 / 56(16.1 \%)$ \\
\hline
\end{tabular}

$\mathrm{AMD}=$ age-related macular degeneration; $\mathrm{BCVA}=$ best-corrected visual acuity; $\mathrm{CNV}=$ choroidal neovascularization; $\mathrm{PDT}=$ photodynamic therapy; PSTA $=$ posterior subtenon injection of triamcinolone acetonide.
Three patients $(6.5 \%)$ suffered from cataract progression and four patients $(8.7 \%)$ suffered from IOP elevation $(>21 \mathrm{mmHg}$ ) in the study group. Among the four patients who suffered from IOP elevation after treatment, three required medical treatment only and one patient needed trabeculectomy in additional to medication. IOP in all four patients returned to normal after treatment. None of the patients had evidence of retinal detachment or endophthalmitis at 12-month follow-up. None of the patients in the control group suffered from cataract progression or IOP elevation.

\section{Discussion}

This study found that PDT combined with PSTA is a more effective therapy than PDT alone in the treatment of subfoveal CNV. At the 12-month follow-up, mean BCVA of patients in the study group improved by 0.78 lines, which is significantly better than that of patients in the control group who had a mean loss of 1.86 lines of BCVA. Furthermore, the difference in number of treatments within 12 months between the study group (mean, 1.26 treatments) and control group (mean, 1.63 treatments) is statistically significant $(P=0.008)$. Therefore, the study indicates that patients who received PDT combined with PSTA had better visual outcome and required fewer treatments. Further analysis showed that the results were obtained mainly from the beneficial response of myopic $\mathrm{CNV}$ and age-related predominantly classic CNV to the combination therapy.

The concept of combination treatment for CNV derives from the understanding of the dynamic processes of $\mathrm{CNV}$ development. By inhibiting CNV growth in different stages, better therapeutic effect may be obtained. ${ }^{14}$ The effect of PDT combined with IVTA for treating $\mathrm{CNV}$ has been evaluated in several studies. Spaide et $a l^{19}$ reported that mean BCVA increased by 2.4 lines in 13 patients who suffered from CNV secondary to AMD 1 year after combined PDT/IVTA treatment. However, Arias et $a l^{18}$ found that there was no significant

Table 5 Treatment outcomes of the study group and the control group in myopic CNV subgroup

\begin{tabular}{lccc}
\hline & PDT + PSTA $(\mathrm{n}=17)$ & PDT alone $(\mathrm{n}=22)$ & P-value \\
\hline Mean baseline BCVA & $0.92( \pm 0.46)$ & $0.9( \pm 0.45)$ & 0.87 \\
Mean number of treatments & $1.18( \pm 0.4)$ & $1.55( \pm 0.74)$ & 0.07 \\
No. of eyes improved $>3$ lines & $5 / 17(29.4 \%)$ & $2 / 22(9.1 \%)$ & 0.11 \\
No. of eyes improved $>2$ lines & $11 / 17(64.7 \%)$ & $4 / 22(18.2 \%)$ & 0.002 \\
No. of eyes improved $>1$ line & $13 / 17(76.5 \%)$ & $7 / 22(31.8 \%)$ & 0.005 \\
No. of eyes lost $>1$ line & $1 / 17(5.9 \%)$ & $9 / 22(40.9 \%)$ & 0.012 \\
No. of eyes lost $>2$ lines & $0 / 17(0 \%)$ & $6 / 22(27.3 \%)$ & 0.019 \\
No. of eyes lost $>3$ lines & $0 / 17(0 \%)$ & $2 / 22(9.1 \%)$ & 0.21 \\
\hline
\end{tabular}

$\mathrm{BCVA}=$ best-corrected visual acuity; $\mathrm{CNV}=$ choroidal neovascularization; $\mathrm{PDT}=$ photodynamic therapy; PSTA $=$ posterior subtenon injection of triamcinolone acetonide. 
difference in mean BCVA change between patients treated with combined PDT/IVTA therapy and those who underwent PDT only. In a study involving 41 patients with occult CNV secondary to AMD, Augustin et $a l^{16}$ reported that mean BCVA increased from 20/133 to 20/84 at 12 months after PDT/IVTA treatment. Furthermore, Ruiz-Moreno et $a l^{17}$ found that BCVA improved by 0.7 Snellen lines in 15 patients with AMD-associated CNV 1 year after they had been treated with PDT combined with IVTA. Most of these studies show that PDT combined with IVTA leads to significant improvement in visual outcome compared with PDT alone in CNV patients. Although comparison between various studies is difficult because of different inclusion criteria and disease entities, our study indicates that mean BCVA change 12 months after treatment with PDT combined with PSTA is as good, if not better than that achieved after treatment with PDT and IVTA.

In addition to providing similar anti-inflammatory and antiangiogenic activity as IVTA, ${ }^{27,28}$ PSTA may be associated with fewer complications than IVTA, as our study indicated. The incidence of IOP elevation $(>21 \mathrm{mmHg}$ ) after the treatment of IVTA has been reported to range from 21 to $43 \% .^{29}$ Although most patients only required medical treatment, surgical intervention was needed in some cases. ${ }^{30,31}$ Cataract progression is another common complication and has been reported to occur in up to $57 \%$ of patients after IVTA. ${ }^{29}$ In a study involving 73 eyes with CNV, Van de Moere $e t a l^{22}$ reported that the incidence of elevated IOP was $7.9 \%$ after the treatment of posterior juxtascleral triamcinolone acetonide. The incidence of IOP elevation and the incidence of cataract progression noted in our study were 8.7 and $6.5 \%$, respectively. The two patients who suffered from cataract progression had the same BCVA 1 year after treatment, indicating improvement of the retina condition. Endophthalmitis is another rare but severe complication of IVTA. ${ }^{29}$ Theoretically, this visionthreatening complication should not occur with a PSTA procedure.

Although general analysis showed better visual outcomes in the study group than in the control group, CNV of different subtypes may respond differently. In TAP and VIP trials, it was reported that the mean change of BCVA 1 year after treatment was +0.2 lines in patients with myopic CNV, -2.2 lines in patients with classic predominant CNV secondary to AMD, and -3.1 lines in patients with occult CNV secondary to AMD. ${ }^{10,11,24}$ Myopic CNV had the best visual outcome among different types of CNV after PDT treatment, and occult CNV secondary to AMD had the poorest outcome. In our study, we also found that visual outcome differed depending on the type of CNV treated. With combination therapy, the proportion of eyes with a BCVA increase of more than three lines in our study was highest in myopic CNV (29.4\%) and 0\% in occult CNV secondary to AMD. In addition, the proportion of eyes with a BCVA loss of more than three lines in the study group was lowest in myopic CNV and highest in occult CNV secondary to AMD. While myopic CNV and age-related predominantly classic $\mathrm{CNV}$ were the two subgroups that responded favourably to combined treatment in the present study, the former seems to gain even greater advantage from this treatment modality than the latter, as the difference of mean BCVA change between monotherapy and combination therapy group was higher in myopic CNV group ( -0.91 to +2.82 lines) than in age-related predominantly classic $\mathrm{CNV}$ group $(-1.79$ to +0.6 lines); the discrepancy of visual acuity gaining more than three lines between the monotherapy and combination therapy groups was also higher in the myopic CNV group. Therefore, among the different types of CNV, PDT combined with PSTA is most effective in treating myopic CNV.

Regarding the treatment of myopic CNV, Marticorena et $a l^{32}$ found a significant increase in mean visual acuity at 1,3, and 6 months after PDT and IVTA; however, high incidence of increased IOP was also noted. Chan et al, ${ }^{33}$ on the other hand, found that there was no significant difference between the treatment of PDT and PDT combined with IVTA. They did find, however, eyes with poorer initial visual acuity (less than 20/80) or larger lesion size (greatest linear dimension $\geq 750 \mu \mathrm{m}$ ) was more likely to gain two or more lines of visual acuity at 1 year with combination treatment. Kojima et $a l^{34}$ found that trans-Tenon's retrobulbar triamcinolone infusion itself would be an effective treatment for myopic CNV. Similar to those results, our study also had good outcomes after the treatment of PDT combined with PSTA for myopic CNV. There are some possible mechanisms that explain why the therapeutic effect of PDT combined with PSTA is better in myopic CNV. First, the thinner sclera of myopic eye may facilitate the diffusion of triamcinolone acetonide from posterior subtenon space to choroid. ${ }^{35}$ Second, the lesion in myopic $\mathrm{CNV}$ is usually small and may be situated in the juxtafoveal site, with haemorrhage or fluid extending to the fovea. As the lesion becomes inactive and subfoveal fluid and haemorrhage reabsorbed after treatment, central vision may recover to a better extent. Third, combination treatment may reduce the incidence of hypertrophic scar or decrease the area and extent of collateral damage that may occur after PDT monotherapy, and may be crucial for visual outcome in high myopic eyes. ${ }^{36}$ In high myopia, the small scar after combined treatment may help better preserve the foveal structure and function. 
The safest and most effective treatment sequence when applying PDT/IVTA therapy has not been determined. ${ }^{15-17,19}$ One of the rationales for performing IVTA after PDT is to avoid the free radical scavenging effect of steroids, since free radicals are important in the mechanism of PDT action. ${ }^{17,37,38}$ Performing PDT followed by IVTA might also prevent the vitreous opacity produced by triamcinolone, which may interfere with the subsequent PDT procedure. ${ }^{17}$ On the other hand, pretreatment with IVTA has the therapeutic advantage of reducing macular oedema or subretinal fluid, making laser delivery to the target tissue more effective. In cases of RPE detachment, performing IVTA first may reduce the risk of RPE tear. ${ }^{39}$ In our study, we performed PSTA after PDT. Care should be taken to avoid exposing the treated eye to bright light to minimize unwanted photosensitizing effects by residual photoporphyrin.

In addition to PDT alone or PDT combined with steroid therapy, many $\mathrm{CNV}$ treatments have been studied, such as monotherapy with anti-VEGF, PDT combined with antiVEGF, and triple therapy with IVTA, PDT, and pegaptanib sodium. ${ }^{40-42}$ In particular, recent studies have shown that repeated monotherapy with anti-VEGF ranibizumab or bevacizumab may improve vision in patients with $\mathrm{CNV}$ of all types. ${ }^{4-45}$ Sakaguchi $e t$ a $l^{46}$ performed intravitreal bevacizumab (1 mg) injection to treat eight eyes with myopic $\mathrm{CNV}$ and improvement of BCVA to two or more lines was found in six eyes (75\%). In our study, myopic $\mathrm{CNV}$ responded markedly better to PDT/PSTA than other types of CNV, and $64.7 \%$ of eyes in this subgroup had improvement of BCVA to more than two lines. Studies designed to compare PDT/PSTA and anti-VEGF therapy in the treatment of myopic CNV may be warranted.

\section{Conclusion}

Although our report was a retrospective study of a small sample of patients from a single medical centre and did not include standard randomization or masking of the investigators, our results were compatible with those reported in large-scale studies that evaluated the treatment of CNV with PDT alone and in combination with IVTA. Of notice, myopic CNV had the best response to the treatment than other types of CNV. To our knowledge, this study is the first to investigate the treatment of different types of CNV with PDT combined with PSTA. A larger randomized trial with a longer follow-up period is suggested to confirm our findings.

\section{References}

1 Green WR, Wilson DJ. Choroidal neovascularization. Ophthalmology 1986; 93: 1169-1176.
2 Klaver CC, Wolfs RC, Vingerling JR, Hofman A, de Jong PT. Age-specific prevalence and causes of blindness and visual impairment in an older population: the Rotterdam Study. Arch Ophthalmol 1998; 116: 653-658.

3 Ferris III FL, Fine SL, Hyman L. Age-related macular degeneration and blindness due to neovascular maculopathy. Arch Ophthalmol 1984; 102: 1640-1642.

4 Miller DG, Singerman LJ. Vision loss in younger patients: a review of choroidal neovascularization. Optom Vis Sci 2006; 83: 316-625.

5 Avila MP, Weiter JJ, Jalkh AE, Trempe CL, Pruett RC, Schepens CL. Natural history of choroidal neovascularization in degenerative myopia. Ophthalmology 1984; 91: 1573-1581.

6 Hampton GR, Kohen D, Bird AC. Visual prognosis of disciform degeneration in myopia. Ophthalmology 1983; 90: 923-926.

7 Wong TY, Foster PJ, Hee J, Ng TP, Tielsch JM, Chew SJ et al. Prevalence and risk factors for refractive errors in adult Chinese in Singapore. Invest Ophthalmol Vis Sci 2000; 41: 2486-2494.

8 Lin LL, Shih YF, Hsiao CK, Chen CJ. Prevalence of myopia in Taiwanese schoolchildren: 1983-2000. Ann Acad Med Singapore 2004; 33: 27-33.

9 Bressler NM, Arnold J, Benchaboune M, Blumenkranz MS, Fish GE, Gragoudas ES et al. Verteporfin therapy of subfoveal choroidal neovascularization in patients with age-related macular degeneration: additional information regarding baseline lesion composition's impact on vision outcomes-TAP report No. 3. Arch Ophthalmol 2002; 120: 1443-1454.

10 Verteporfin in Photodynamic Therapy Study Group. Verteporfin therapy of subfoveal choroidal neovascularization in age-related macular degeneration: two-year results of a randomized clinical trial including lesions with occult with no classic choroidal neovascularization - verteporfin in photodynamic therapy report 2. Am J Ophthalmol 2001; 131: 541-560.

11 Verteporfin in Photodynamic Therapy Study Group. Photodynamic therapy of subfoveal choroidal neovascularization in pathologic myopia with verteporfin. 1-year results of a randomized clinical trial-VIP report no. 1. Ophthalmology 2001; 108: 841-852.

12 Verteporfin Roundtable Participants. Guidelines for using verteporfin (Visudyne) in photodynamic therapy for choroidal neovascularization due to age-related macular degeneration and other causes: update. Retina 2005; 25 : 119-134.

13 Schmidt-Erfurth U, Schlötzer-Schrehard U, Cursiefen C, Michels S, Beckendorf A, Naumann GO. Influence of photodynamic therapy on expression of vascular endothelial growth factor (VEGF), VEGF receptor 3, and pigment epithelium-derived factor. Invest Ophthalmol Vis Sci 2003; 44: 4473-4480.

14 Grossniklaus HE, Green WR. Choroidal neovascularization. Am J Ophthalmol 2004; 137: 496-503.

15 Augustin AJ, Schmidt-Erfurth U. Verteporfin therapy combined with intravitreal triamcinolone in all types of choroidal neovascularization due to age-related macular degeneration. Ophthalmology 2006; 11: 14-22.

16 Augustin AJ, Schmidt-Erfurth U. Verteporfin and intravitreal triamcinolone acetonide combination therapy for occult choroidal neovascularization in age-related macular degeneration. Am J Ophthalmol 2006; 141: 638-645. 
17 Ruiz-Moreno JM, Montero JA, Barile S, Zarbin MA. Photodynamic therapy and high-dose intravitreal triamcinolone to treat exudative age-related macular degeneration: 1-year outcome. Retina 2006; 26: 602-612.

18 Arias L, Garcia-Arumi J, Ramon JM, Badia M, Rubio M, Pujol O. Photodynamic therapy with intravitreal triamcinolone in predominantly classic choroidal neovascularization: one-year results of a randomized study. Ophthalmology 2006; 113: 2243-2250.

19 Spaide RF, Sorenson J, Maranan L. Photodynamic therapy with verteporfin combined with intravitreal injection of triamcinolone acetonide for choroidal neovascularization. Ophthalmology 2005; 112: 301-304.

20 Zarbin M. Should corticosteroids be considered as part of the standard care with photodynamic therapy? Arch Ophthalmol 2006; 124: 563-571.

21 Helm CJ, Holland GN. The effects of posterior subtenon injection of triamcinolone acetonide in patients with intermediate uveitis. Am J Ophthalmol 1995; 120: 55-64.

22 Van de Moere A, Sandhu SS, Kak R, Mitchell KW, Talks SJ. Effect of posterior juxtascleral triamcinolone acetonide on choroidal neovascular growth after photodynamic therapy with verteporfin. Ophthalmology 2005; 112: 1896-1903.

23 Mueller AJ, Jian G, Banker AS, Rahhal FM, Capparelli E, Freeman WR. The effect of deep posterior subtenon injection of corticosteroids on intraocular pressure. Am J Ophthalmol 1998; 125: 158-163.

24 Treatment of Age-Related Macular Degeneration with Photodynamic Therapy (TAP) Study Group. Photodynamic therapy of subfoveal choroidal neovascularization in age-related macular degeneration with verteporfin: one-year results of 2 randomized clinical trials-TAP report. Arch Ophthalmol 1999; 117: 1329-1345.

25 Bakri SJ, Kaiser PK. Posterior subtenon triamcinolone acetonide for refractory diabetic macular edema. Am J Ophthalmol 2005; 139: 290-294.

26 Neovascular Age-Related Macular Degeneration, Periocular Corticosteroids and Photodynamic Therapy (NAPP) Trial Research Group. Periocular triamcinolone and photodynamic therapy for subfoveal choroidal neovascularization in age-related macular degeneration. Ophthalmology 2007; 114: 1713-1721.

27 Ciulla TA, Criswell MH, Danis RP, Hill TE. Intravitreal triamcinolone acetonide inhibits choroidal neovascularization in a laser-treated rat model. Arch Ophthalmol 2001; 119: 399-404.

28 Ciulla TA, Criswell MH, Danis RP, Fronheiser M, Yuan P, Cox TA et al. Choroidal neovascular membrane inhibition in a laser treated rat model with intraocular sustained release triamcinolone acetonide microimplants. Br J Ophthalmol 2003; 87: 1032-1037.

29 Schmidt-Erfurth U, Michels S, Augustin A. Perspectives on verteporfin therapy combined with intravitreal corticosteroids. Arch Ophthalmol 2006; 124: 561-563.

30 Agrawal S, Agrawal J, Agrawal TP. Vitrectomy as a treatment for elevated intraocular pressure following intravitreal injection of triamcinolone acetonide. Am J Ophthalmol 2004; 138: 679-680.

31 Jonas JB, Akkoyun I, Budde WM, Kreissig I, Degenring RF. Intravitreal reinjection of triamcinolone for exudative age-related macular degeneration. Arch Ophthalmol 2004; 122: 218-222.

32 Marticorena J, Gomez-Ulla F, Fernandez M, Pazos B, Rodriguez-Cid MJ, Sanchez-Salorio M. Combined photodynamic therapy and intravitreal triamcinolone acetonide for the treatment of myopic subfoveal choroidal neovascularization. Am J Ophthalmol 2006; 142: 335-337.

33 Chan WM, Lai TY, Wong AL, Liu DT, Lam DS. Combined photodynamic therapy and intravitreal triamcinolone injection for the treatment of choroidal neovascularisation secondary to pathological myopia: a pilot study. $\mathrm{Br}$ J Ophthalmol 2007; 91: 174-179.

34 Kojima A, Ohno-Matsui K, Futagami S, Shimada N, Tokoro T, Mochizuki M. Trans-Tenon's retrobulbar triamcinolone infusion for myopic choroidal neovascularization. Acta Ophthalmol Scand 2006; 84: 749-754.

35 Rada JA, Shelton S, Norton TT. The sclera and myopia. Exp Eye Res 2006; 82: 185-200.

36 Ruiz-Moreno JM, Montero JA. Subretinal fibrosis after photodynamic therapy in subfoveal choroidal neovascularisation in highly myopic eyes. $\mathrm{Br} J$ Ophthalmol 2003; 87: 856-859.

37 Fukushima K, Ando M, Ito K, Suga M, Araki S. Stimulusand cumulative dose-dependent inhibition of $\mathrm{O}_{2-}$ production by polymorphonuclear leukocytes of patients receiving corticosteroids. J Clin Lab Immunol 1990; 33: 117-123.

38 Weishaupt KR, Gomer CJ, Dougherty TJ. Identification of singlet oxygen as the cytotoxic agent in photoinactivation of a murine tumor. Cancer Res 1976; 36: 2326-2329.

39 Sutter FK, Kurz-Levin MM, Scherrer M, Barthelmes D, Fleischhauer JC, Helbig H. Intravitreal triamcinolone acetonide for serous retinal pigment epithelial detachments in exudative age-related macular degeneration. Klin Monatsbl Augenheilkd 2007; 224: 297-299.

40 Liggett PE, Colina J, Chaudhry NA, Tom D, Haffner G. Triple therapy of intravitreal triamcinolone, photodynamic therapy, and pegaptanib sodium for choroidal neovascularization. Am J Ophthalmol 2006; 142: 1072-1074.

41 Augustin AJ, Puls S, Offermann I. Triple therapy for choroidal neovascularization due to age-related macular degeneration: verteporfin PDT, bevacizumab, and dexamethasone. Retina 2007; 27: 133-140.

42 Dhalla MS, Shah GK, Blinder KJ, Ryan EH, Mittra RA, Tewari A. Combined photodynamic therapy with verteporfin and intravitreal bevacizumab for choroidal neovascularization in age-related macular degeneration. Retina 2006; 26: 988-993.

43 Rosenfeld PJ, Brown DM, Heier JS, Boyer DS, Kaiser PK, Chung CY et al. Ranibizumab for neovascular age-related macular degeneration. N Engl J Med 2006; 355: 1419-1431.

44 Narayanan R, Kuppermann BD, Jones C, Kirkpatrick P. Ranibizumab. Nat Rev Drug Discov 2006; 5: 815-816.

45 Emerson MV, Lauer AK, Flaxel CJ, Wilson DJ, Francis PJ, Stout JT et al. Intravitreal bevacizumab (Avastin) treatment of neovascular age-related macular degeneration. Retina 2007; 27: 439-444.

46 Sakaguchi H, Ikuno Y, Gomi F, Kamei M, Sawa M, Tsujikawa $\mathrm{M}$ et al. Intravitreal injection of bevacizumab for choroidal neovascularisation associated with pathological myopia. Br J Ophthalmol 2007; 91: 161-165. 